\title{
Highly Elevated Serum Hepcidin in Patients with Acute Myeloid Leukemia prior to and after Allogeneic Hematopoietic Cell Transplantation: Does This Protect from Excessive Parenchymal Iron Loading?
}

\author{
Ann-Kathrin Eisfeld, ${ }^{1}$ Mark Westerman, ${ }^{2}$ Rainer Krahl, ${ }^{1}$ Sabine Leiblein, ${ }^{1}$ \\ Uwe Gerd Liebert, ${ }^{3}$ Marianne Hehme, ${ }^{4}$ Daniel Teupser, ${ }^{5}$ \\ Dietger Niederwieser, ${ }^{1}$ and Haifa Kathrin Al-Ali ${ }^{1}$ \\ ${ }^{1}$ Department of Hematology/Oncology, University of Leipzig, Johannesallee 32a, 04103 Leipzig, Germany \\ ${ }^{2}$ Intrinsic LifeSciences LLC, La Jolla, CA 92037, USA \\ ${ }^{3}$ Institute of Virology, University of Leipzig, 04103 Leipzig, Germany \\ ${ }^{4}$ Novartis Pharma GmbH, 90429 Nuernberg, Germany \\ ${ }^{5}$ Institute of Laboratory Medicine, University of Leipzig, 04103 Leipzig, Germany
}

Correspondence should be addressed to Haifa Kathrin Al-Ali, alah@medizin.uni-leipzig.de

Received 30 December 2010; Accepted 7 March 2011

Academic Editor: J. F. San Miguel

Copyright (C) 2011 Ann-Kathrin Eisfeld et al. This is an open access article distributed under the Creative Commons Attribution License, which permits unrestricted use, distribution, and reproduction in any medium, provided the original work is properly cited.

Hepcidin is upregulated by inflammation and iron. Inherited (HFE genotype) and treatment-related factors (blood units (BU), Iron overload) affecting hepcidin (measured by C-ELISA) were studied in 42 consecutive patients with AML prior to and after allogeneic hematopoietic cell transplantation (HCT). Results. Elevated serum ferritin pre- and post-HCT was present in all patients. Median hepcidin pre- and post-HCT of 358 and $398 \mathrm{ng} / \mathrm{mL}$, respectively, were elevated compared to controls (median $52 \mathrm{ng} / \mathrm{mL})(P<.0001)$. Liver and renal function, prior chemotherapies, and conditioning had no impact on hepcidin. Despite higher total BU after HCT compared to pretransplantation $(P<.0005)$, pre- and posttransplant ferritin and hepcidin were similar. BU influenced ferritin $(P=.001)$ and hepcidin $(P=.001)$. No correlation of pre- or posttransplant hepcidin with pretransplant ferritin was found. HFE genotype did not influence hepcidin. Conclusions. Hepcidin is elevated in AML patients pre- and post-HCT due to transfusional iron-loading suggesting that hepcidin synthesis remains intact despite chemotherapy and HCT.

\section{Introduction}

Hepcidin, a 25-amino acid antimicrobial peptide synthesized in the liver, is the central iron-regulatory hormone that mediates the homeostasis of extracellular iron concentrations [ 1 , 2]. Studies have confirmed its role as the negative regulator of iron absorption, recycling, and release from stores. Hepcidin regulates iron export into plasma from tissues involved in iron storage or transport such as duodenal enterocytes, hepatocytes, macrophages, and the syncytial trophoblasts of the placenta [3]. Hepcidin binds to the iron exporter ferroportin and induces its internalization and degradation.
Systemic iron requirements and infectious as well as inflammatory stimuli modulate hepcidin expression $[4,5]$. Its transcription is upregulated by cytokines such as interleukin6 and interleukin-1 as well as by iron and is downregulated by iron deficiency, ineffective erythropoiesis, and hypoxia $[4,6]$. Because of its sensitivity to inflammatory stimuli and its effect on iron export from various tissues, hepcidin plays an essential role for iron disorders such as anemia of inflammation [7]. Hepcidin expression is also associated with transfusional iron overload in $\beta$-thalassemia and other iron-loading anemias [8]. 
Additionally, evidence indicates that the hemochromatosis gene HFE might be required for the synthesis of hepcidin. Recent studies with animal models of hemochromatosis suggest an important role of HFE protein as an iron sensor and upstream regulator of hepcidin in hepatocytes. Hepatocyte HFE is necessary for signaling to hepcidin, presumably as a constituent of a larger iron-sensing complex [9-12].

Iron overload, mainly due to multiple blood transfusions, is a common complication in recipients of hematopoietic cell transplantation (HCT) [13]. Iron overload increases the risk of infections, veno-occlusive disease, and hepatic dysfunction after HCT. An increase of pretransplant serum ferritin levels, which usually reflects body iron stores, has been shown to lower disease-free survival following HCT and might increase the risk of graft-versus-host disease (GvHD). However, additional studies are needed to determine the influence of iron overload on long-term morbidity and mortality in allogeneic HCT survivors [14].

In patients with acute myeloid leukemia (AML) receiving HCT, both inherited and treatment-related factors might influence hepcidin expression. Whether hepcidin synthesis and regulation remain intact despite intensive AML-chemotherapy and HCT is yet unknown. Therefore, the impact of pretransplant body iron load, blood transfusions, HFE genotype as well as chemotherapy, conditioning regimen, and graft-versus-host disease (GVHD) on serum hepcidin levels was analyzed in patients with AML prior to and after allogeneic HCT.

\section{Materials and Methods}

2.1. Study Design. Between February 2008 and February 2009, 42 consecutive patients with AML in complete remission who received allogeneic HCT at the University of Leipzig, Germany, and survived three months were included. All patients were assessed clinically and biochemically on two occasions: ten days prior to and at a median of 3 (range 35) months after allogeneic HCT. All medications including antibiotics, antifungal, and immunosuppressive drugs were recorded. The study was conducted in accordance with the Declaration of Helsinki [15]. Patients gave written informed consent.

2.2. Patients. Median age was 57 (range 18-70) years. 23 (54.8\%) were male, and $19(45.2 \%)$ patients were female. Median number of AML-chemotherapies prior to HCT was 3 (range, 0-5). Conventional conditioning with 12 Gray total body irradiation (TBI) and cyclophosphamide $120 \mathrm{mg} / \mathrm{kg}$ was given to $13(31 \%)$ patients while $29(69 \%)$ of patients received HCT following reduced intensity conditioning (RIC) with fludarabin $30 \mathrm{mg} / \mathrm{m}^{2} /$ day for 3 days and 2 Gray TBI applied once followed by immunosuppression with cyclosporine (through level $\geq 200 \mathrm{ng} / \mathrm{mL}$ ) and mycophenolate mofetil. Donors were matched related (MRD) in $8(19 \%)$ and matched unrelated (MUD) in 34 (81\%) patients. Acute GVHD confirmed by biopsy of at least one involved site was graded according to the Glucksberg-Seattle criteria [16]. $14(33.3 \%)$ patients developed grade $\geq$ II acute GVHD of
TABLE 1: Patient characteristics at baseline $(n=42)$.

\begin{tabular}{|c|c|}
\hline Parameters & Results \\
\hline Mean age, years (range) & $57(18-70)$ \\
\hline \multicolumn{2}{|l|}{ Gender, $n(\%)$} \\
\hline Male & $23(54.8)$ \\
\hline Female & $19(45.2)$ \\
\hline \multicolumn{2}{|l|}{ Donor, $n(\%)$} \\
\hline MRD & $8(19 \%)$ \\
\hline MUD & $34(81 \%)$ \\
\hline \multicolumn{2}{|l|}{ Conditioning, $n(\%)$} \\
\hline Conventional & $13(31.0)$ \\
\hline RIC & $29(69.0)$ \\
\hline \multicolumn{2}{|l|}{ Acute GVHD, $n(\%)$} \\
\hline \multicolumn{2}{|l|}{ Skin } \\
\hline No & $7(16.7)$ \\
\hline Yes & $35(83.3)$ \\
\hline Grade I & 21 \\
\hline Grade II & 10 \\
\hline Grade III & 4 \\
\hline \multicolumn{2}{|l|}{ Liver } \\
\hline No & $35(83.3)$ \\
\hline Yes & $7(16.7)$ \\
\hline Grade I & 6 \\
\hline Grade II & 1 \\
\hline Grade III & 0 \\
\hline \multicolumn{2}{|c|}{ Median number of blood transfusions (range) } \\
\hline Prior to HCT & $22(8-95)$ \\
\hline After HCT & $30(14-120)$ \\
\hline \multicolumn{2}{|c|}{ Median serum ferritin (range) $\mathrm{ng} / \mathrm{mL}$} \\
\hline Prior to HCT & $1945(617-6981)$ \\
\hline After HCT & $2260(807-7595)$ \\
\hline \multicolumn{2}{|c|}{ Median serum hepcidin (range) $\mathrm{ng} / \mathrm{mL}$} \\
\hline Prior to HCT & $358(56-1096)$ \\
\hline After HCT & $398(172-941)$ \\
\hline
\end{tabular}

MRD: matched related donor; MUD: matched unrelated donor; RIC: reduced intensity conditioning; GVHD: graft versus host disease; HCT: hematopoietic stem cell transplantation.

the skin while $7(16.7 \%)$ patients suffered grade $\geq$ II acute GVHD of the liver.

Patients received a median of 22 (range 8-95) units of blood pre-HCT and a median total of 30 (14-120) units after HCT. Table 1 summarises patient characteristics.

2.3. Body Iron. For assessment of body iron stores, serum ferritin was measured. At the time of assessment, patients were in a stable clinical condition without signs of infection and a concomitant C-reactive protein (CRP) $<10 \mathrm{mg} / \mathrm{L}$. Normal reference value for ferritin was $30-400 \mathrm{ng} / \mathrm{mL}$.

2.4. Hepcidin. Concomitant with the measurement of serum ferritin, serum hepcidin was measured by a hepcidin competitive enzyme-linked immunoassay (C-ELISA) at Intrinsic LifeSciences LLC, La Jolla, CA, USA, as previously described 
[17]. In healthy volunteers, the $5 \%$ to $95 \%$ range of hepcidin concentrations was $29-254 \mathrm{ng} / \mathrm{mL}$ for males $(n=65)$ and $17-286 \mathrm{ng} / \mathrm{mL}$ for females $(n=49)$ with median concentrations of 112 and $65 \mathrm{ng} / \mathrm{mL}$, respectively $(P<.001)$ [17].

In this study, serum hepcidin concentrations of 21 healthy volunteers matched for age and gender (6 male, 15 female, median age 57 years) were used as controls.

2.5. HFE Gene Analysis. Patients were screened for their HFE genotype prior to and after HCT. Mutations in DNA extracted from peripheral blood were detected by polymerase chain reaction (PCR) using hybridization probes and melting curve analysis as described previously [18].

2.6. Donor Cell Chimerism. After conventional conditioning, donor chimerism was analysed in unsorted bone marrow cells day 28 after HCT. For all patients who received RIC HCT, additionally flow-sorted T (CD3+)-, and CD34+-bone marrow cells at days $28,56,84$, and at 3 months interval thereafter was monitored by fluorescence in situ hybridisation (FISH) for the XY chromosome in gender mismatched or PCR-based analysis of polymorphic microsatellite regions in gender-matched HCT. All patients expressed a full donor chimerism after HCT.

2.7. Liver and Renal Function. Alanine aminotransferase (ALT), aspartate aminotransferase (AST), alkaline phosphates (AP), and bilirubin were evaluated regularly. Normal laboratory reference value for ALT/AST, AP, and bilirubin were $\leq 0.6 \mathrm{mmoL} / \mathrm{L}, \leq 1.74 \mu \mathrm{kat} / \mathrm{L}$, and $\leq 17 \mu \mathrm{moL} / \mathrm{L}$, respectively. Creatinine was used to monitor renal function. Normal reference value for creatinine was $<104 \mu \mathrm{moL} / \mathrm{L}$ (Table 1).

2.8. Statistical Analysis. For statistical analysis Wilcoxon signed-rank test and Mann-Whitney $U$ test were used. CoxRegression was used for multivariate analysis. For nonparametric correlations, we used the Spearman-Rho-Test and the Pearson-correlation. Data were analyzed using SPSS15 software. A $P$ value of $<.05$ was considered statistically significant.

\section{Results}

3.1. Body Iron Stores prior to and after HCT. Prior to allogeneic HCT, excess body iron was present in all patients with a median serum ferritin of 1945 (range 617-6981) $\mathrm{ng} / \mathrm{mL}$. Patients received a median of 22 (range 8-95) units of blood. After HCT, the median serum ferritin of 2260 (range $807-7595) \mathrm{ng} / \mathrm{mL}$ was not statistically higher than the pretransplant level although the number of blood transfusions after HCT reached a median of 30 (range 14-120) units which was significantly more than that received prior to HCT $(P<.0005)$. The number of blood transfusions prior to HCT strongly correlated with the pretransplant serum ferritin $(P=.001)$. Again, the posttransplant serum ferritin

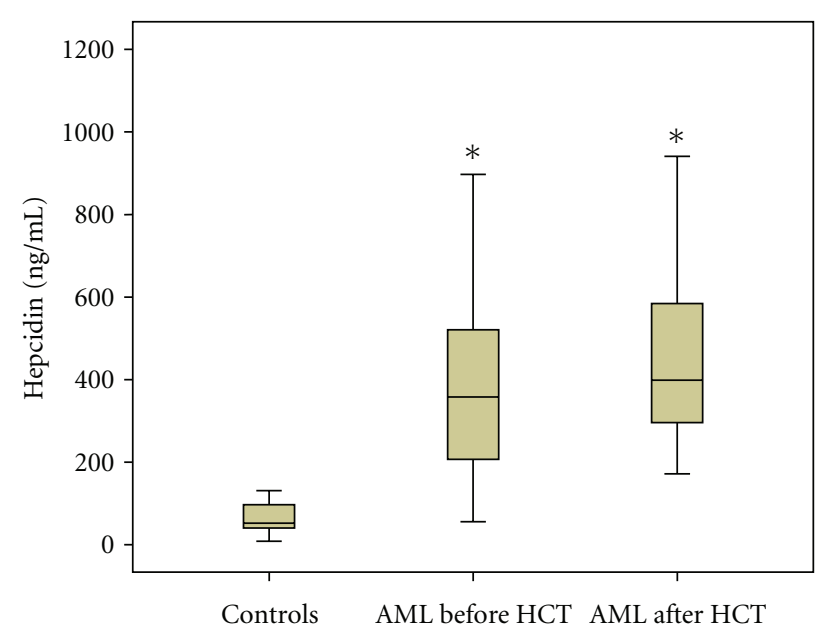

FIGURE 1: Hepcidin concentrations in controls and in AML patients before and after allogeneic HCT $(n=42) ;{ }^{*} P<.0001$, each versus controls.

was strongly dependent on the cumulative number of blood units transfused $(P<.001)$.

3.2. HFE Genotypes prior to and after HCT. Data on HFE gene status were available in 41 patients prior to and in 40 patients after HCT. In the pre-transplantation setting, mutations were found in 19 (46.0\%) patients. The most frequently occurring HFE mutation was heterozygosity (het) for H63D $(n=11)$, followed by het C282Y $(n=3)$, and het S65C $(n=1)$. Homozygosity (homo) for H63D was detected in 4 patients. After HCT, HFE mutations were detected in 15 (37.5\%) patients. Again heterozygosity for H63D occurred most frequently $(n=12)$, followed by het S65C $(n=2)$, and het $\mathrm{C} 282 \mathrm{Y}(n=1)$. Compound-het was detected in one patient after allogeneic HCT. Interestingly, in 18 patients the HFE genotype changed after HCT (Table 2). HFE genotype at any time point had no influence on serum ferritin.

3.3. Hemoglobin $(\mathrm{Hb})$ prior to and after HCT. Prior to HCT, $\mathrm{Hb}$ with a median of 10.3 (range 6.8-14.5) g/dL was similar to Hb levels after HCT (median 10.1 (range 6.7-13.4) g/dL). There was a negative correlation between $\mathrm{Hb}$ levels and serum ferritin prior to HCT $(P=.002)$. Similarly, higher ferritin values were associated with lower $\mathrm{Hb}$ levels after transplantation $(P=.01)$.

3.4. Hepcidin Levels prior to and after HCT. The median hepcidin concentration of 52.1 (range 8.3-130.8) ng/mL in the 21 healthy volunteers in this analysis was comparable to the published values in healthy volunteers measured with this assay [17].

Patients with AML demonstrated highly elevated serum hepcidin levels both in the pre-transplant (median 358 (range 56-1096) ng/mL) and post-transplant setting (median 398 (range 172-941) ng/mL) compared to the control group $(P<.0001)$. There was no statistically significant 
TABLE 2: HFE mutations before and after allogeneic HCT.

\begin{tabular}{|c|c|c|}
\hline Patient ID & $\begin{array}{l}\text { HFE mutations } \\
\text { before HCT }\end{array}$ & $\begin{array}{c}\text { HFE mutations } \\
\text { after HCT }\end{array}$ \\
\hline 1 & heth63D & wt \\
\hline 2 & homoH63D & wt \\
\hline 3 & wt & wt \\
\hline 4 & wt & wt \\
\hline 5 & wt & heth63D \\
\hline 6 & wt & wt \\
\hline 7 & wt & wt \\
\hline 8 & wt & wt \\
\hline 9 & wt & heth63D \\
\hline 10 & wt & wt \\
\hline 11 & hetC282Y & wt \\
\hline 12 & wt & wt \\
\hline 13 & heth63D & -1 \\
\hline 14 & wt & wt \\
\hline 15 & hetC282Y & wt \\
\hline 16 & wt & wt \\
\hline 17 & wt & wt \\
\hline 18 & hetH63D & hetH63D \\
\hline 19 & wt & wt \\
\hline 20 & hetH63D & wt \\
\hline 21 & hetC282Y & wt \\
\hline 22 & hetH63D & hetH63D \\
\hline 23 & wt & hetH63D \\
\hline 24 & hetH63D & hetH63D \\
\hline 25 & hetH63D & hetH63D \\
\hline 26 & hetH63D & hetH63D \\
\hline 27 & wt & wt \\
\hline 28 & wt & wt \\
\hline 29 & hetH63D & hetS65C \\
\hline 30 & homoH63D & wt \\
\hline 31 & homoH63D & wt \\
\hline 32 & hetS65C & wt \\
\hline 33 & wt & hetH63D \\
\hline 34 & wt & hetH63D \\
\hline 35 & homoH63D & wt \\
\hline 36 & hetH63D & hetS65C \\
\hline 37 & hetH63D & hetH63D \\
\hline 38 & wt & comp. het \\
\hline 39 & wt & wt \\
\hline 40 & wt & wt \\
\hline 41 & wt & hetH63D \\
\hline 42 & -1 & -1 \\
\hline
\end{tabular}

HFE: hereditary hemochromatosis gene; HCT: hematopoietic cell transplantation; het: heterozygous; homo: homozygous; comp. het: compound heterozygous; wt: wildtype.

difference between median hepcidin levels prior to and after HCT $(P=.4)$ (Figure 1$)$.
3.4.1. Correlation of Hepcidin Levels with Blood Transfusions. Hepcidin levels before HCT strongly correlated with the number of blood transfusions prior to HCT $(P=.001)$ (Figure 2(a)). Similarly, post-transplant hepcidin values significantly correlated with both the number of blood units transfused prior to transplantation $(P=.008)$ and the total units of blood transfused $(P<.001)$.

3.4.2. Correlation of Hepcidin Levels with Body Iron. Surprisingly in the pre-transplant setting, and although the number of blood units transfused strongly correlated with the serum ferritin, no statistical correlation was found between serum ferritin values prior to HCT and serum hepcidin concentrations prior to or after transplantation (Figure 2(b)).

3.4.3. Correlation of Serum Hepcidin with Hb Levels. Hepcidin concentrations significantly correlated inversely with Hb values $(P=.002)$ (Figure $2(\mathrm{c}))$. A similar association could be found between hepcidin and $\mathrm{Hb}$ values after HCT $(P=.001)$.

3.4.4. Effect of HFE Genotype on Hepcidin Levels. In our cohort, HFE genotype prior to or after HCT had no impact on hepcidin values at any time point.

3.4.5. Effect of Other Patient Characteristics on Hepcidin Levels. In univariate analysis, age and gender had no significant influence on hepcidin values before or after HCT. Similarly, liver and renal functions, number of prior chemotherapies, and type of conditioning regimen had no impact on hepcidin levels either before or after HCT.

3.5. Outcome after HCT. After a median followup of 21 (range 17-28) months, the probability of survival and leukemia-free survival (LFS) at 2 years were $66 \%$ and $64 \%$, respectively. The incidences of relapse and nonrelapse mortality (NRM) at 2 years were $20 \%$. In this analysis, pre- and post-HCT serum ferritin and hepcidin levels as well as the number of blood transfusions did not correlate with survival, LFS, relapse, or NRM. Acute graft-versushost disease (GVHD) which occurred in $35(83 \%)$ patients was limited to acute cutaneous GVHD stage I and II in 31 patients. Chronic GVHD (limited, $n=4$, extensive, $n=18)$ was present in $22(53 \%)$ patients. Again, there was no association between the occurrence and the severity of GVHD on one side and pre- or post-HCT body iron or hepcidin levels on the other side. In the first 100 days post-HCT, 12 (29\%) patients suffered a nonlethal grade 3 infection according to common toxicity criteria. In the entire cohort, serum ferritin or hepcidin values did not influence the occurrence of severe infections.

\section{Discussion}

Evidence for iron overload as determined by serum ferritin concentrations was found in all polytransfused AML patients. Serum ferritin is a practical measure of body iron 


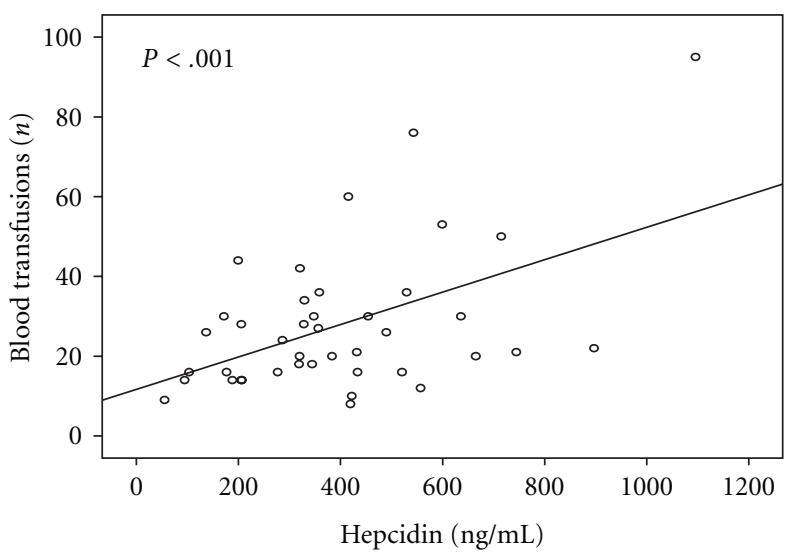

(a)

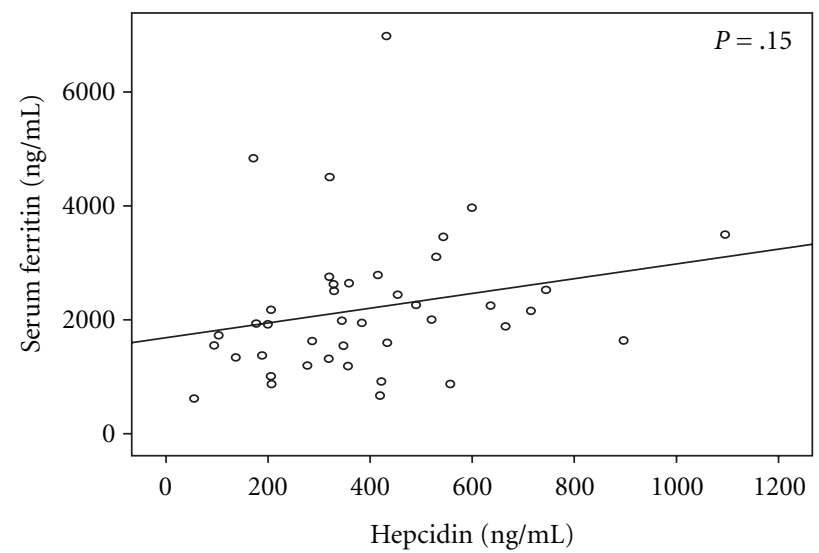

(b)

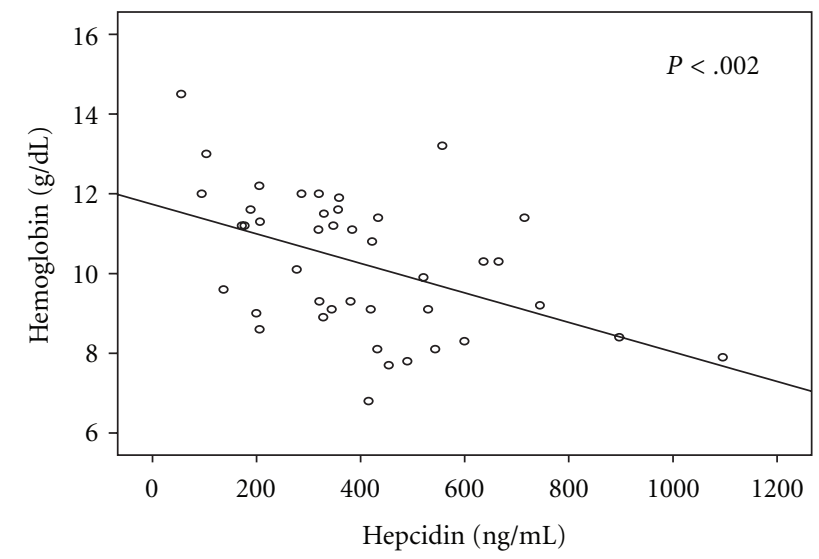

(c)

Figure 2: Correlation of serum hepcidin levels with number of blood transfusions (a), serum ferritin values (b), and hemoglobin levels (c) before HCT.

that can easily be applied and is supposed to represent a reliable marker of body iron stores [19-21]. Although factors such as infection, acute and chronic inflammation, and alcohol abuse can exert an influence on individual values, both the stable clinical condition and the concomitant low CRP in all patients suggest that the serum ferritin mainly reflected body iron stores.

It is not surprising that serum ferritin at all time points strongly correlated with the number of blood transfusions as iron overload, commonly observed in recipients of allogeneic HCT, is mainly attributed to multiple blood transfusions. But other factors as overexpression of the growth and differentiation factor (GDF-15) which inhibits the production of hepcidin in the liver have been discussed as possible contributors to excess body iron in thalassemia [22].

In this study, patients with AML compared to healthy volunteers demonstrated highly elevated serum hepcidin levels strongly correlating with the number of blood transfusions both in the pre- and post-transplant setting. This finding suggests that transfusional iron remains a dominant regulator of hepcidin in these patients and that hepcidin synthesis and regulation remain intact in polytransfused patients with AML undergoing allogeneic HCT irrespective of the number of prior chemotherapies, type of conditioning regimen, or antifungal treatments.

Usually, hepcidin and serum ferritin respond similarly to inflammation and changes in iron stores, and this is reflected in the strong correlation between hepcidin and ferritin in healthy volunteers with hepcidin responses taking place on the time scale of a few hours, whereas changes in ferritin concentrations are much slower [23]. However, no correlation of pre- or post-HCT hepcidin levels with serum ferritin values prior to HCT was found despite the fact that both serum ferritin and hepcidin were strongly affected by the number of blood units transfused. Also, pre- and post-transplant serum ferritin and hepcidin values were statistically similar despite significantly higher cumulative blood units received after HCT. These data suggest that hepcidin represents a compensatory mechanism that counteracts transfusional iron input. Hepcidin is known to bind to ferroportin, leading to intracellular retention of iron in macrophages and to a reduction of extracellular iron bound to transferrin or serum ferritin [24]. This raises the fascinating question of whether elevated hepcidin values in the presence of transfusional iron loading might protect from excessive parenchymal iron overload and subsequent organ damage. 
An elevated hepcidin expression as a proposed cause of anemia of chronic inflammation [7] might explain the inverse correlation of hepcidin levels with $\mathrm{Hb}$ values observed in this study and highlight the complexity of hepcidin regulation.

As HFE gene mutations have a high frequency in population of European descent [9-11], patients in this study were screened prior to and after HCT for HFE gene mutations to determine whether HFE genotype contributed to excess iron and to assess the impact of HFE gene mutations on hepcidin concentrations. As all patients after HCT expressed full donor chimerism, the HFE genotype after HCT must be considered to reflect donor genotype. HFE deletions or mutations have been associated with a reduction of liver hepcidin expression [12, 25-27]. However, it is presently controversial whether HFE is indispensable for hepcidin activation in response to inflammatory stimuli [28-30]. Mutations of HFE account for most but not all cases of hemochromatosis [31]. The most common mutation, C282Y, is associated with disruption of a disulfide bond in HFE that is critical for its binding to $\beta_{2}$ microglobulin [32]. This interaction is required for the stabilisation, transport, and expression of HFE on the cell surface and endosomal membranes where HFE interacts with the transferrin receptor 1 (TfR1). The H63D mutation, a common HFE mutation whose pathogenic significance is still uncertain, does not impair interaction between HFE and TfR1. As the H63D HFE mutation was the only mutation commonly detected in our cohort, this might in part explain why HFE genotype had no influence on serum hepcidin levels.

Similarly, the missing association between HFE genotype and serum ferritin in our cohort is in accordance with published data. Veneri et al. studied the prevalence of 12 mutations of the HFE gene and its correlation with the iron status in 82 adult patients with acute leukemia of whom 58.5\% were affected by acute myeloid leukemia (AML) [33]. $32.9 \%$ of the patients had at least one HFE gene mutation and mean serum ferritin levels were increased at diagnosis. However, there was no difference between patients positive or negative for the HFE mutations in terms of serum ferritin levels. The study therefore highlighted the presence of iron overload in many AML patients but did not support the evidence of an association between HFE mutations and iron overload in acute leukemia [33].

Increased pre-transplant iron overload has been demonstrated to represent negative prognostic factors in patients with myelodysplastic syndromes or secondary AML [34], thalassemia, and other hematologic disorders such as acute leukemias $[35,36]$ receiving HCT irrespective of the type of conditioning [35-37].

The impact of an elevated hepcidin level on outcome after HCT is not yet known. But few data suggest that pretransplant serum hepcidin levels might predict the risk of early infectious bacterial complications after allogeneic HCT [38].

Although the aim of this work was not to study the impact of hepcidin values on outcome after HCT, nevertheless no negative impact of an elevated pre-transplant hepcidin concentration on outcome, the incidence or severity of acute or chronic GVHD, or early infectious complications after HCT could be detected in this cohort. It is important to keep in mind that this was a small series of patients surviving the first three months after HCT. Nevertheless, this observation merits further investigation with a higher number of patients.

\section{Conclusions}

For the first time, we describe highly elevated serum hepcidin concentrations in AML patients with transfusional iron load both prior to and after HCT using the C-ELISA for human serum hepcidin assessment. This suggests that hepcidin synthesis and upregulation remain intact despite intensive chemotherapy and HCT. The weak correlation between pretransplant serum ferritin and both pre- or post-transplant serum hepcidin is highly interesting although both preand post-transplant levels of serum ferritin and hepcidin were similar and correlated, as would be expected, with the number of blood units received. So Overexpression of hepcidin might represent a compensatory mechanism counteracting transfusional iron input.

Actually, overexpression of hepcidin might play an important protective role in this setting as it might prevent an increased ferroportin-mediated iron export from macrophages thereby reducing the severity of parenchymal iron loading and damage. In contrast to ferritin, a marker of iron stores, changes in hepcidin concentrations are frequently the cause of iron disorders. Thus, measurements of hepcidin concentrations in addition to serum ferritin might be highly informative in understanding iron homeostasis in the setting of HCT. To establish the exact impact of serum hepcidin on outcome after HCT, large prospective studies are warranted.

\section{Conflict of Interests}

Hepcidin measurement was financed with a grant from Novartis, Germany.

\section{Acknowledgment}

The authors would like to thank Dr. Joachim Sauer for the editorial assistance provided during preparation of this paper.

\section{References}

[1] G. Nicolas, M. Bennoun, I. Devaux et al., "Lack of hepcidin gene expression and severe tissue iron overload in upstream stimulatory factor 2 (USF2) knockout mice," Proceedings of the National Academy of Sciences of the United States of America, vol. 98, no. 15, pp. 8780-8785, 2001.

[2] M. V. Verga Falzacappa and M. U. Muckenthaler, "Hepcidin: Iron-hormone and anti-microbial peptide," Gene, vol. 364, no. 1-2, pp. 37-44, 2005.

[3] A. S. Zhang and C. A. Enns, "Molecular mechanisms of normal iron homeostasis," Hematology / The Education Program of the American Society of Hematology, pp. 207-214, 2009. 
[4] C. Pigeon, G. Ilyin, B. Courselaud et al., "A new mouse liver-specific gene, encoding a protein homologous to human antimicrobial peptide hepcidin, is overexpressed during iron overload," Journal of Biological Chemistry, vol. 276, no. 11, pp. 7811-7819, 2001.

[5] L. Lin, E. V. Valore, E. Nemeth, J. B. Goodnough, V. Gabayan, and T. Ganz, "Iron transferrin regulates hepcidin synthesis in primary hepatocyte culture through hemojuvelin and BMP2/4," Blood, vol. 110, no. 6, pp. 2182-2189, 2007.

[6] E. Nemeth, E. V. Valore, M. Territo, G. Schiller, A. Lichtenstein, and T. Ganz, "Hepcidin, a putative mediator of anemia of inflammation, is a type II acute-phase protein," Blood, vol. 101, no. 7, pp. 2461-2463, 2003.

[7] T. Ganz, "Hepcidin, a key regulator of iron metabolism and mediator of anemia of inflammation," Blood, vol. 102, no. 3, pp. 783-788, 2003.

[8] E. Nemeth and T. Ganz, "Hepcidin and iron-loading anemias," Haematologica, vol. 91, no. 6, pp. 727-732, 2006.

[9] O. K. Fix and K. V. Kowdley, "Hereditary hemochromatosis," Minerva Medica, vol. 99, no. 6, pp. 605-617, 2008.

[10] E. H. Hanson, G. Imperatore, and W. Burke, "HFE gene and hereditary hemochromatosis: a HuGE review," American Journal of Epidemiology, vol. 154, no. 3, pp. 193-206, 2001.

[11] P. Pedersen and N. Milman, "Genetic screening for HFE hemochromatosis in 6,020 Danish men: penetrance of C282Y, H63D, and S65C variants," Annals of Hematology, vol. 88, no. 8, pp. 775-784, 2009.

[12] K. Pantopoulos, "Function of the hemochromatosis protein HFE: lessons from animal models," World Journal of Gastroenterology, vol. 14, no. 45, pp. 6893-6901, 2008.

[13] N. S. Majhail, H. M. Lazarus, and L. J. Burns, "Iron overload in hematopoietic cell transplantation," Bone Marrow Transplantation, vol. 41, no. 12, pp. 997-1003, 2008.

[14] N. S. Majhail, T. DeFor, H. M. Lazarus, and L. J. Burns, "High prevalence of iron overload in adult allogeneic hematopoietic cell transplant survivors," Biology of Blood and Marrow Transplantation, vol. 14, no. 7, pp. 790-794, 2008.

[15] World Medical Association, "Declaration of Helsinki: ethical principles for medical research involving human subjects," Journal of International Biotechnology Law, vol. 15, pp. 124129, 2004.

[16] H. Glucksberg, R. Storb, and A. Fefer, "Clinical manifestations of graft versus host disease in human recipients of marrow from HL A matched sibling donors," Transplantation, vol. 18, no. 4, pp. 295-304, 1974.

[17] T. Ganz, G. Olbina, D. Girelli, E. Nemeth, and M. Westerman, "Immunoassay for human serum hepcidin," Blood, vol. 112, no. 10, pp. 4292-4297, 2008.

[18] E. Lyon, "Mutation detection using fluorescent hybridization probes and melting curve analysis," Expert Review of Molecular Diagnostics, vol. 1, no. 1, pp. 92-101, 2001.

[19] J. M. Bennett, "Consensus statement on iron overload in myelodysplastic syndromes," American Journal of Hematology, vol. 83, no. 11, pp. 858-861, 2008.

[20] N. Gattermann, "Guidelines on iron chelation therapy in patients with myelodysplastic syndromes and transfusional iron overload," Leukemia Research, vol. 31, no. 3, pp. S10-S15, 2007.

[21] A. Piperno, "Classification and diagnosis of Iron overload," Haematologica, vol. 83, no. 5, pp. 447-455, 1998.

[22] T. Tanno, N. V. Bhanu, P. A. Oneal et al., "High levels of GDF15 in thalassemia suppress expression of the iron regulatory protein hepcidin," Nature Medicine, vol. 13, no. 9, pp. 10961101, 2007.
[23] E. Kemna, P. Pickkers, E. Nemeth, H. Van Der Hoeven, and D. Swinkels, "Time-course analysis of hepcidin, serum iron, and plasma cytokine levels in humans injected with LPS," Blood, vol. 106, no. 5, pp. 1864-1866, 2005.

[24] M. U. Muckenthaler, "Fine tuning of hepcidin expression by positive and negative regulators," Cell Metabolism, vol. 8, no. 1, pp. 1-3, 2008.

[25] B. A. C. Van Dijk, C. M. M. Laarakkers, S. M. Klaver et al., "Serum hepcidin levels are innately low in HFE-related haemochromatosis but differ between C282Y-homozygotes with elevated and normal ferritin levels," British Journal of Haematology, vol. 142, no. 6, pp. 979-985, 2008.

[26] A. Piperno, D. Girelli, E. Nemeth et al., "Blunted hepcidin response to oral iron challenge in HFE-related hemochromatosis," Blood, vol. 110, no. 12, pp. 4096-4100, 2007.

[27] D. F. Wallace, L. Summerville, E. M. Crampton, D. M. Frazer, G. J. Anderson, and V. N. Subramaniam, "Combined deletion of Hfe and transferrin receptor 2 in mice leads to marked dysregulation of hepcidin and iron overload," Hepatology, vol. 50, no. 6, pp. 1992-2000, 2009.

[28] P. Lee, H. Peng, T. Gelbart, and E. Beutler, "The IL-6and lipopolysaccharide-induced transcription of hepcidin in HFE-, transferrin receptor 2-, and $\beta$-microglobulin-deficient hepatocytes," Proceedings of the National Academy of Sciences of the United States of America, vol. 101, no. 25, pp. 9263-9265, 2004.

[29] C. N. Roy, Á. O. Custodio, J. De Graaf et al., "An Hfedependent pathway mediates hyposideremia in response to lipopolysaccharide-induced inflammation in mice," Nature Genetics, vol. 36, no. 5, pp. 481-485, 2004.

[30] D. M. Frazer, S. J. Wilkins, K. N. Millard, A. T. McKie, C. D. Vulpe, and G. J. Anderson, "Increased hepcidin expression and hypoferraemia associated with an acute phase response are not affected by inactivation of HFE," British Journal of Haematology, vol. 126, no. 3, pp. 434-436, 2004.

[31] A. Pietrangelo, "Hemochromatosis 1998: is one gene enough?" Journal of Hepatology, vol. 29, no. 3, pp. 502-509, 1998.

[32] A. Waheed, S. Parkkila, X. Y. Zhou et al., "Hereditary hemochromatosis: effects of C282Y and H63D mutations on association with $\beta 2$-microglobulin, intracellular processing, and cell surface expression of the HFE protein in COS-7 cells," Proceedings of the National Academy of Sciences of the United States of America, vol. 94, no. 23, pp. 12384-12389, 1997.

[33] D. Veneri, M. Franchini, M. Krampera, G. De Matteis, P. Solero, and G. Pizzolo, "Analysis of HFE and TFR2 gene mutations in patients with acute leukemia," Leukemia Research, vol. 29, no. 6, pp. 661-664, 2005.

[34] Z. Y. Lim, V. Fiaccadori, S. Gandhi et al., "Impact of pre-transplant serum ferritin on outcomes of patients with myelodysplastic syndromes or secondary acute myeloid leukaemia receiving reduced intensity conditioning allogeneic haematopoietic stem cell transplantation," Leukemia Research, vol. 34, no. 6, pp. 723-727, 2010.

[35] A. Mahindra, B. Bolwell, R. Sobecks et al., "Elevated pretransplant ferritin is associated with a lower incidence of chronic graft-versus-host disease and inferior survival after myeloablative allogeneic haematopoietic stem cell transplantation," British Journal of Haematology, vol. 146, no. 3, pp. 310-316, 2009.

[36] K. Kataoka, Y. Nannya, A. Hangaishi et al., "Influence of pretransplantation serum ferritin on nonrelapse mortality after myeloablative and nonmyeloablative allogeneic hematopoietic stem cell transplantation," Biology of Blood and Marrow Transplantation, vol. 15, no. 2, pp. 195-204, 2009. 
[37] V. Pullarkat, S. Blanchard, B. Tegtmeier et al., "Iron overload adversely affects outcome of allogeneic hematopoietic cell transplantation," Bone Marrow Transplantation, vol. 42, no. 12, pp. 799-805, 2008.

[38] J. Kanda, C. Mizumoto, H. Kawabata et al., "Clinical significance of serum hepcidin levels on early infectious complications in allogeneic hematopoietic stem cell transplantation," Biology of Blood and Marrow Transplantation, vol. 15, no. 8, pp. 956-962, 2009. 


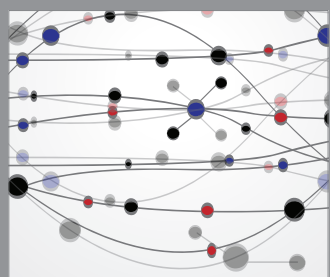

The Scientific World Journal
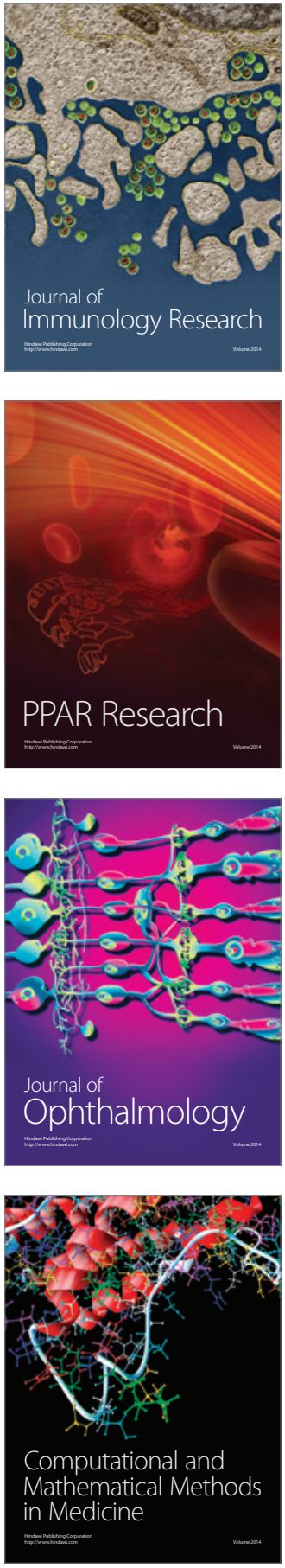

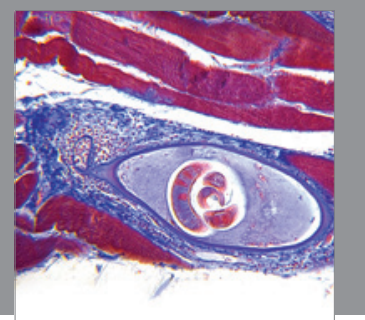

Gastroenterology

Research and Practice
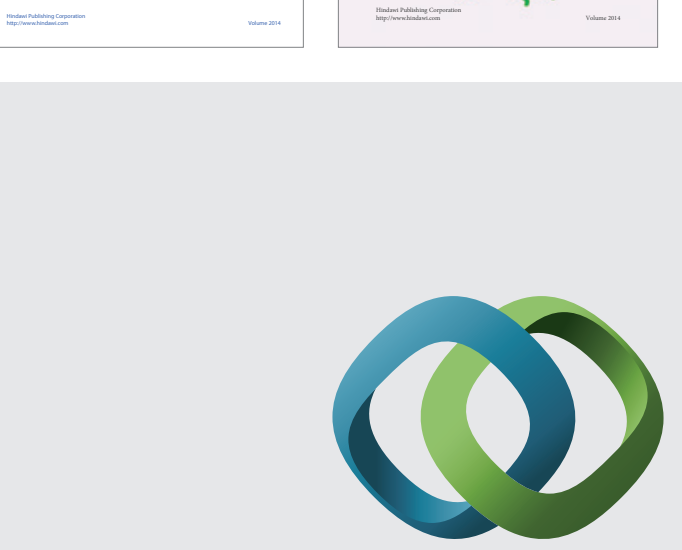

\section{Hindawi}

Submit your manuscripts at

http://www.hindawi.com
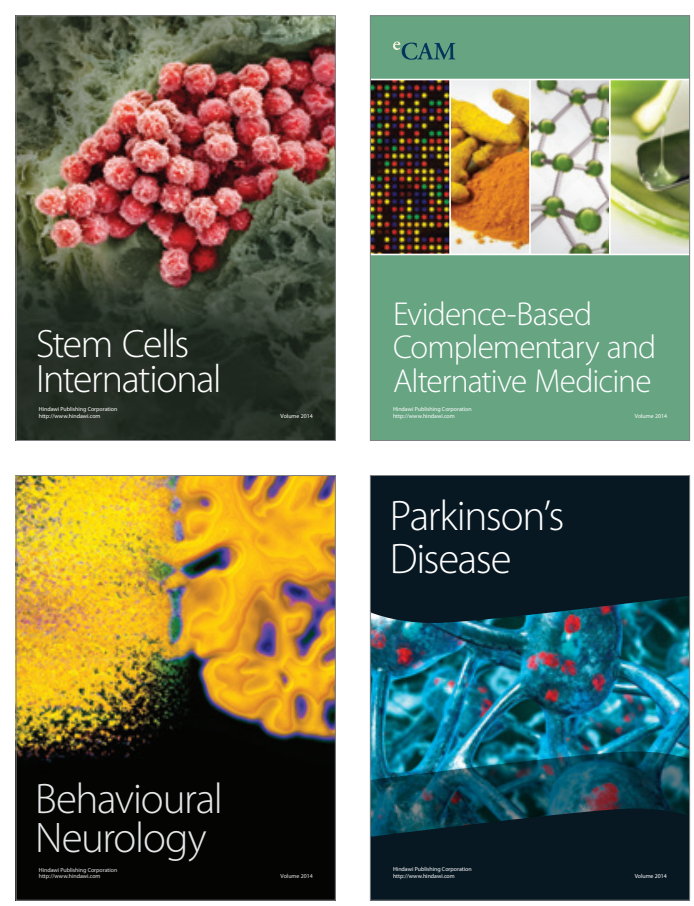

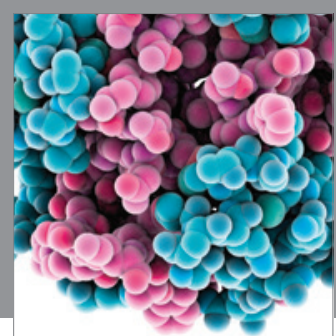

Journal of
Diabetes Research

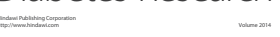

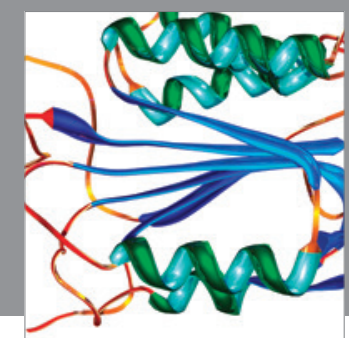

Disease Markers
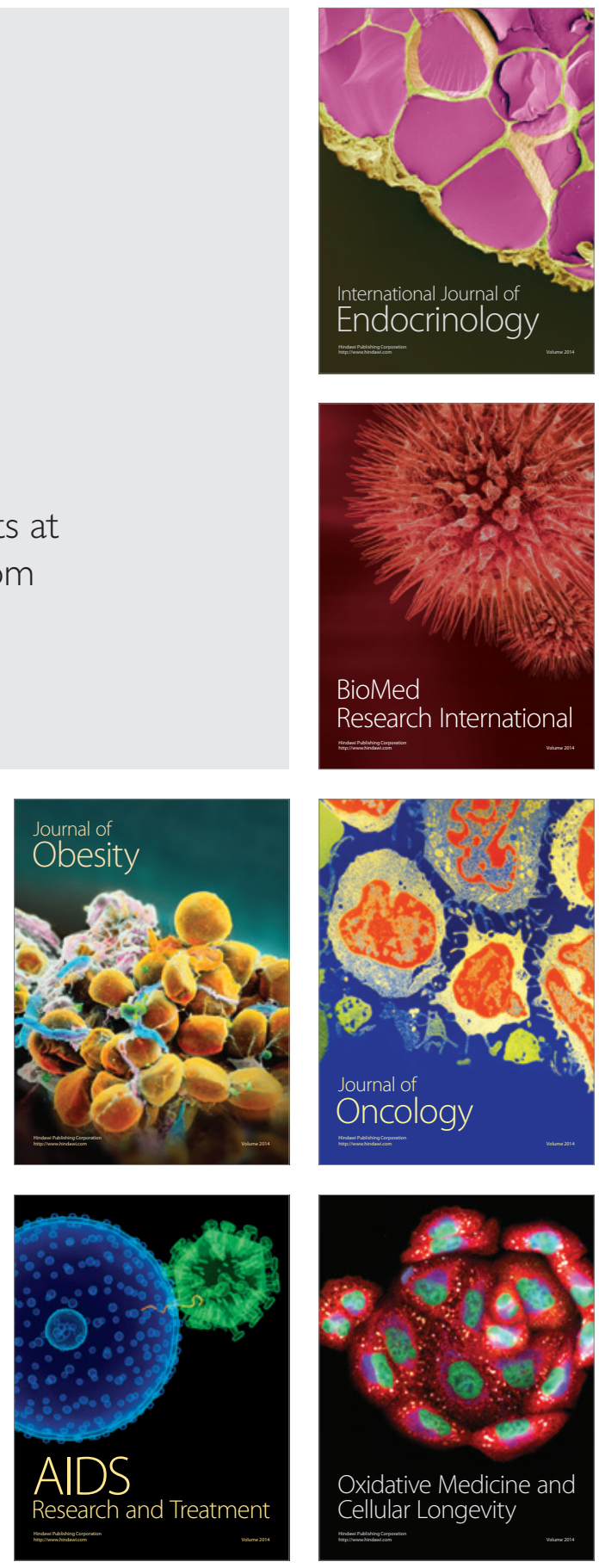